\title{
Enseñanzas y aprendizajes sobre la Cátedra de la paz en Colombia*
}

Catalina María Vásquez-Russi

https://orcid.org/oooo-0001-5943-9171

Universidad Nacional de Colombia,

sede Medellín, Colombia

cmvasquezr@unal.edu.co

\section{Resumen}

La Cátedra de la Paz es un espacio académico obligatorio para todas las instituciones educativas de Colombia, que tiene como objetivo generar competencias ciudadanas para una convivencia democrática, respetuosa de los Derechos Humanos y en paz. Este artículo analiza la producción bibliográfica sobre la Cátedra de la Paz a partir de tres categorías: cómo se define la paz, qué rol se les asigna a las instituciones educativas en la formación de ciudadanías de paz y cuáles son los enfoques y metodologías de enseñanza para la aplicación de los principios y contenidos de la Cátedra. Se concluye que la bibliografía sobre la Cátedra de la Paz debe profundizar en el estudio de imaginarios, posibilidades y caracteristicas de los estudiantes, para crear rutas de acción más certeras sobre la formación de ciudadanos para la paz. Por otro lado, se encontró un consenso sobre el rol de los colegios como responsables principales de la formación ciudadana, teniendo como punto de partida la participación de los estudiantes.

\section{Palabras clave (Fuente: tesauro de la Unesco)}

Cátedra de la paz; Colombia; currículum escolar; educación escolar; educación para la paz.
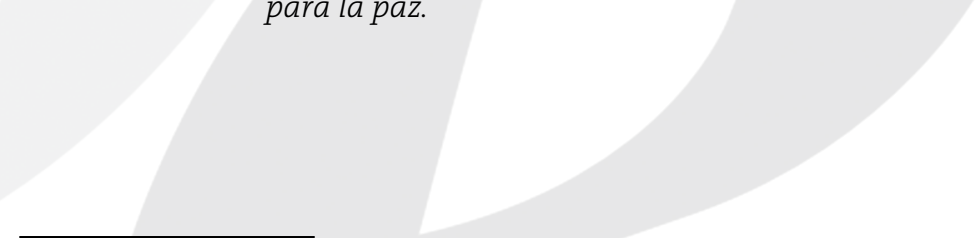
* Este trabajo fue financiado por el Ministerio de Ciencia Tecnología e Innovación y la Universidad Nacional de
Colombia, sede Medellín, a través de la convocatoria Joven Investigador Colciencias 812 de 2018.

Recepción: 21/01/2020 | Envío a pares: 05/03/2020 | Aceptación por pares: 20/03/2020 | Aprobación: 27/05/2020 


\title{
Lessons and Takeaways from the Lecture on Peace in Colombia
}

\begin{abstract}
The Lecture on Peace (Cátedra para la paz) is a compulsory subject for all educational institutions in Colombia whose aim is to develop citizen skills for democratic, peaceful, human rights-oriented coexistence. This article analyzes literature production on the Lecture on Peace from three categories: how peace is defined, what role is assigned to educational institutions in the training of peace-making citizens, and what the teaching approaches and methods are for the application of the principles and contents of the lecture. It concludes that such literature must delve into the study of students' imaginary, possibilities, and characteristics to devise more accurate action plans on the training of peace-making citizens. Besides, a consensus was found on the role of schools as the parties responsible for citizen education, taking students' participation as a starting point.
\end{abstract}

Keywords (Source: Unesco thesaurus)

Chair for peace; Colombia; school curriculum; school education; peace education. 


\section{Ensino e aprendizagem sobre a "cadeira da paz" na Colômbia}

Resumo

A "cadeira da paz" é um espaço acadêmico obrigatório para todas as instituições educativas da Colômbia. Tem o objetivo de gerar competências cidadãs para uma convivência democrática, respeitosa dos Direitos Humanos e em paz. Este artigo analisa a produção bibliográfica sobre essa disciplina a partir de três categorias: como a paz é definida; que papel é designado às instituições educativas na formação de cidadanias de paz e quais são as abordagens e metodologias de ensino para aplicar os princípios e conteúdo da disciplina. Este artigo conclui que a bibliografia deve aprofundar no estudo de imaginários, possibilidades e características dos estudantes, para criar estratégias de ação mais exatas sobre a formação de cidadãos para a paz. Por sua vez, verificou-se que um consenso sobre o papel dos colégios como responsáveis principais da formação cidadã, tendo como ponto de partida a participação dos estudantes.

\section{Palavras-chave (Fonte: tesauro da Unesco)}

cadeira da paz; Colômbia; currículo escolar; educação escolar; educação para a paz. 
La Cátedra de la Paz es un espacio académico obligatorio para todas las instituciones educativas del país. Creado por la Ley 1732 de 2014 del Congreso de la República y reglamentado por el Decreto 1038 de 2015 , su objetivo es "fomentar el proceso de apropiación de conocimientos y competencias relacionados con el territorio, la cultura, el contexto económico y social y la memoria histórica, con propósito de reconstruir el tejido social, promover la prosperidad general y garantizar la efectividad de los principios, derechos y deberes consagrados en la Constitución" (Presidencia de la República, 2015)'.

Los principios rectores de la Cátedra de la Paz son el reconocimiento del otro, la formación de pensamiento crítico, el conocimiento de sí y la construcción de democracia, lo cual implica una atención especial a los contenidos y a la construcción de una metodología de aprendizaje que sea democrática en sí misma. La Cátedra de la Paz puede ser implementada como asignatura separada o incluida en los Planes de Asignatura del Área de Ciencias Sociales o áreas afines, lo cual lleva a cuestionamientos sobre su aplicación real en el aula y las posibilidades de evaluación de sus competencias. A su vez, se pone en duda la necesidad de su existencia, al ya tener implementados los Estándares Básicos de Competencias en Ciencias Sociales y los Estándares Básicos de Competencias Ciudadanas, del Ministerio de Educación Nacional (2004a/b).

En 2018, 9.916.546 alumnos fueron matriculados en la educación formal preescolar, básica primaria, básica secundaria, media y en los Ciclos Lectivos Especiales Integrados (CLEI) en Colombia. De estos, el 80,4\% pertenecían al sector oficial y 19,6\%

Cada institución del país debe desarrollar, al menos, dos de los siguientes componentes: Justicia y Derechos Humanos, Uso sostenible de los recursos naturales, Protección de las riquezas culturales y naturales de la Nación, Resolución pacífica de conflictos, Prevención del acoso escolar, Diversidad y pluralidad, Participación Política, Memoria histórica, Dilemas morales, Proyectos de impacto social, Historia de los acuerdos de paz nacionales e internacionales y Proyectos de vida y prevención de riesgos (Presidencia de la República, 2015). al sector no oficial, según el Departamento Administrativo Nacional de Estadística (DANE, 2018). Esto significa que, teóricamente, $22,55 \%$ de la población de Colombia ${ }^{2}$ se ve impactada por los contenidos de la Cátedra de la Paz3. Si se asume que el propósito fundamental de esta es "garantizar la creación y el fortalecimiento de una cultura de paz en Colombia", es claro que la responsabilidad de las instituciones educativas es enorme. De ahí surge la necesidad de indagar por los avances académicos en referencia a la Cátedra de la Paz.

En esa línea, la presente revisión documental analiza la producción bibliográfica sobre la enseñanza de la Cátedra de la Paz, desde tres perspectivas: cómo ha definido la paz, qué rol se les asigna a las instituciones educativas en la formación de ciudadanos y cuáles son las metodologías de enseñanza que permitirían la aplicación de los principios y contenidos de la Cátedra. Para ello, se evalúan fuentes escritas primarias y secundarias, oficiales y no oficiales, legales y académicas, referentes a la Cátedra de Paz entre 2014 y 2019, incluyendo información del Ministerio de Educación, el Sistema Nacional de Bibliotecas (SINAB) de la Universidad Nacional de Colombia, e información de las bases de datos Scielo, Redalyc y Google Scholar, así como libros y otras publicaciones oficiales. Es importante aclarar que sobre este tema no ha habido una producción bibliográfica significativa en el ámbito pedagógico y académico.

El artículo se divide en cuatro secciones: primero se comentan críticamente los conceptos de "paz" manejados por la literatura sobre la Cátedra; luego, se analiza el rol asignado a las instituciones

2 En este porcentaje se tienen en cuenta solo los estudiantes de educación formal preescolar, básica primaria, básica secundaria, media y CLEI (Ciclos Lectivos Especiales Integrados), excluyendo a los estudiantes de educación superior, a los cuales también se aplica la Ley 1475 de 2014 y el Decreto 1038 de 2015.

3 El Departamento Administrativo Nacional de Estadística (DANE) estimó que Colombia contó en 2018 con una población total de 48.258 .494 (Herrera, 2019). 
educativas en el siglo XXI en la formación de ciudadanías; en tercer lugar, se ahonda en los enfoques pedagógicos y metodologías de enseñanza de los contenidos de la Cátedra y, finalmente, se realiza un análisis propositivo sobre los jóvenes en Colombia y sus posibilidades y limitaciones. Todos los apartados contienen una reflexión crítica que permite identificar avances y dificultades teóricas y prácticas de la educación para la paz en Colombia.

\section{La paz: un concepto en disputa}

La Cátedra de la Paz surgió en un contexto de transformación de la política de seguridad del Estado colombiano, en la cual se priorizó el diálogo sobre la confrontación armada. Así, entre 2012 y 2016, el Estado llevó a cabo negociaciones de paz con las Fuerzas Armadas Revolucionarias de Colombia (FARCEP). En este contexto de negociación, la paz supuso el cese permanente de la guerra entre los dos frentes. Sin embargo, la coyuntura política permitió también un análisis sobre los orígenes de la guerra en Colombia y sus consecuencias en la cultura de los ciudadanos, que concluyó que los colombianos tendrían dificultades para resolver como sociedad sus disputas políticas, ideológicas, sociales, culturales, etc., de forma pacífica. Esto pudo evidenciarse en las guerras decimonónicas entre conservadores y liberales, hasta los magnicidios del siglo XX (Jorge Eliécer Gaitán, Luis Carlos Galán, monseñor Isaías Duarte, Jaime Garzón, Rodrigo Lara, entre otros), así como en el mismo surgimiento de las guerrillas de izquierda y los grupos paramilitares de derecha.

Así, con el "Acuerdo Final para la terminación del conflicto y la construcción de una paz estable y duradera", se hacía necesario fomentar no solo el conocimiento sobre la historia de Colombia, sino, principalmente, el desarrollo de habilidades para la vida que supusieran una transformación de la cultura política nacional desde los primeros años de vida. En la firma del Decreto 1038 de 2015 "por el cual se reglamenta la Cátedra de la Paz", el entonces presidente de Colombia, Juan Manuel Santos, afirmó lo siguiente:
En los últimos años, se ha venido repitiendo un mismo titular en los medios de comunicación: "En Colombia, las riñas dejan más muertos que la guerra". Es increíble, pero cierto. La violencia no es ajena al diario vivir de los colombianos, y es por eso que debemos trabajar en los diferentes sectores de la sociedad; no sólo en la Habana, para hacer una nación más tolerante, para que nuestras discusiones no las resolvamos con agresiones. Ser el país mejor educado de América Latina, como nos lo hemos propuesto en tan sólo una década, significa también que seamos un país más pacifico, más tolerante. Hoy damos un paso adelante en este propósito, con la firma del Decreto que reglamenta la ley de la Cátedra de la Paz. En virtud de este decreto, colegios y universidades del país, comenzarán a impartir esta cátedra en los próximos meses. La idea es que nuestros niños y jóvenes aprendan principios y valores básicos, en asuntos como la reconciliación, la solución amigable de los problemas, un tema muy importante, el respeto por los Derechos Humanos. Si llevamos más de cincuenta años en guerra, no cabe la menor duda de que llegó el momento de educar para la paz, de formar para la paz, de enseñar a vivir en paz. Y ahi, la comunidad educativa puede, debe ser, la protagonista. (Editorial Santillana, 2015, $\min$ 14:09 a 16:12)

El objetivo de la Ley 1732 de 2014 "por medio de la cual se establece la Cátedra de la Paz en todas las instituciones educativas del país" es "crear y consolidar un espacio para el aprendizaje, la reflexión y el diálogo sobre la cultura de la paz y el desarrollo sostenible que contribuya al bienestar general y el mejoramiento de la calidad de vida de la población" (Congreso de la República, 2014), lo cual implica necesariamente una discusión sobre el significado de la palabra paz. Las definiciones de la paz y la educación para la paz que trabajan los estudios sobre la Cátedra pueden ser agrupadas en tres líneas conceptuales: la paz según el sociólogo noruego Johan Galtung, la paz según la Organización de las Naciones Unidas (ONU) y la paz como un derecho y un deber constitucionales. 
La preocupación por la paz y las posibilidades de llegar a ella a través de la educación surgen a partir de la Segunda Guerra Mundial de la necesidad histórica de crear espacios de reconocimiento entre actores diversos, para garantizar la no repetición del genocidio judío y de los enfrentamientos nacionalistas. La paz como prioridad en la agenda política mundial fue la causa y la consecuencia de la creación de la Organización de Naciones Unidas (ONU). Su carta de fundación fue firmada el 26 de junio de 1945 en San Francisco, Estados Unidos, y afirma lo siguiente en su artículo 1:

Los Propósitos de las Naciones Unidas son: 1. Mantener la paz y la seguridad internacionales, $y$ con tal fin: tomar medidas colectivas eficaces para prevenir y eliminar amenazas a la paz, y para suprimir actos de agresión u otros quebrantamientos de la paz; y lograr por medios pacificos, y de conformidad con los principios de la justicia y del derecho internacional, el ajuste o arreglo de controversias o situaciones internacionales susceptibles de conducir a quebrantamientos de la paz; 2. Fomentar entre las naciones relaciones de amistad basadas en el respeto al principio de la igualdad de derechos y al de la libre determinación de los pueblos, y tomar otras medidas adecuadas para fortalecer la paz universal; 3. Realizar la cooperación internacional en la solución de problemas internacionales de carácter económico, social, cultural o humanitario, y en el desarrollo y estímulo del respeto a los derechos humanos y a las libertades fundamentales de todos, sin hacer distinción por motivos de raza, sexo, idioma o religión; y 4. Servir de centro que armonice los esfuerzos de las naciones por alcanzar estos propósitos comunes. $(1945, p .3)$

Así, en documentos expedidos por la Asamblea General de la ONU, como la Declaración Universal de los Derechos Humanos, se afirma:

La educación tendrá por objeto el pleno desarrollo de la personalidad humana y el fortale- cimiento del respeto a los derechos humanos y a las libertades fundamentales; favorecerá la comprensión, la tolerancia y la amistad entre todas las naciones y todos los grupos étnicos o religiosos, y promoverá el desarrollo de las actividades de las Naciones Unidas para el mantenimiento de la paz. (ONU, 1948)

Desde la mitad del siglo XX hasta la actualidad se ha partido de la premisa de que la paz está relacionada con la ausencia de guerra, pero también con la construcción de sociedades resilientes, tolerantes y con capacidad de resolver pacíficamente todo conflicto social. Por tanto, se ha asumido que estas habilidades debían ser enseñadas intencionalmente a todos los niveles de la sociedad, pero, especialmente, desde la temprana infancia. En este contexto, el sociólogo noruego Johan Galtung comienza a publicar varios estudios sobre su teoría de los conflictos y en su primera publicación sobre el tema introduce los conceptos de paz positiva y paz negativa:

One may now look upon peace research as research into the conditions for moving closer to the state we have called GCP [General and complete peace], or at least not drifting closer towards GCW [General and complete war]. Thus, there are two aspects of peace as conceived of here: negative peace which is the absence of violence, absence of war - and positive peace which is the integration of human society. Correspondingly, there are two branches of peace research. $(1964$, p. 2)

Esta conceptualización es el punto de partida de muchos autores y estudios sobre la Cátedra de la Paz en Colombia. En concreto, Pachón y Garzón afirman que la paz, "definida en sentido positivo, es un estado a nivel social o personal, en el cual se encuentran en equilibrio y estabilidad las partes de una unidad; definida en sentido negativo, es la ausencia de inquietud, violencia o guerra. En el plano colectivo 'paz' es lo contrario de la guerra estado interior [...] exento de sentimientos negativos (ira, odio)" (2016, 
p. 41). Esta misma perspectiva es compartida por Carmona (2016) y Forero (2018). A su vez, es explicada en detalle por Tejeda y Del Pozo (2016) y Díaz et al. (2016). Sin embargo, es Salas (2017, p. 42) quien profundiza en la tipología de Galtung, al dividirla en violencia directa (daño físico o psicológico directo), violencia estructural (represión o explotación) y violencia cultural o simbólica (en la educación, la música, el arte, el lenguaje) . Los conceptos de Galtung son desarrollados ampliamente en la bibliografía sobre la Cátedra de la Paz, sin embargo, no son adecuados al contexto escolar.

Un acercamiento a cómo estos conceptos son aplicados en la vida diaria dentro de una institución educativa aparece en el documento "Orientaciones de la implementación de la Cátedra de Paz con enfoque de cultura ciudadana" expedido por la Alcaldía Mayor de Bogotá, el cual afirma que "en el escenario escolar, estas dos dimensiones de la paz se traducen en que no haya agresión ni maltrato y en que se promuevan la inclusión, la no discriminación, el balance de poder y la equidad. Lo que hacen las competencias ciudadanas es fortalecer los dos tipos de paz, especialmente en el ámbito convivencia y paz" (2018, p. 26). En sintesis, el primer abordaje sobre el concepto de paz y educación para la paz de la literatura existente entiende que la paz es la ausencia de violencia (sea esta directa, estructural, cultural o simbólica) y requiere la construcción de relaciones armoniosas en comunidad (Romero, 2010).

La segunda perspectiva sobre la paz hallada en la bibliografía parte de lo estipulado por la Organización de las Naciones Unidas (ONU), específicamente en su Resolución A RES/53/243 del 6 de octubre de 1999, en la cual se define el concepto de "cultura de paz" como:

...conjunto de valores, actitudes, tradiciones, comportamientos y estilos de vida basados en: a) El respeto a la vida, el fin de la violencia y la promoción y la práctica de la no violencia por medio de la educación, el diálogo y la cooperación; b) El respeto pleno de los principios de soberania, integridad territorial e independencia política de los Estados y de no injerencia en los asuntos que son esencialmente jurisdicción interna de los Estados, de conformidad con la Carta de las Naciones Unidas y el derecho internacional; c) El respeto pleno y la promoción de todos los derechos humanos y las libertades fundamentales; d) El compromiso con el arreglo pacífico de los conflictos; e) Los esfuerzos para satisfacer las necesidades de desarrollo y protección del medio ambiente de las generaciones presente y futuras; f) El respeto y la promoción del derecho al desarrollo; g) El respeto y el fomento de la igualdad de derechos y oportunidades de mujeres y hombres; h) El respeto y el fomento del derecho de todas las personas a la libertad de expresión, opinión e información; i) La adhesión a los principios de libertad, justicia, democracia, tolerancia, solidaridad, cooperación, pluralismo, diversidad cultural, diálogo y entendimiento a todos los niveles de la sociedad y entre las naciones; $y$ animados por un entorno nacional e internacional que favorezca a la paz. (Asamblea General, 1999)

Esta perspectiva difiere de la de Galtung en tanto profundiza en los valores, actitudes, principios y comportamientos que sustentan la idea de la paz en la sociedad y ahonda en los retos de formación de futuros ciudadanos. Así, la visión de la ONU se presenta menos concreta, pero más profunda en todas las implicaciones sociales de la búsqueda de una cultura de paz. Esta visión es compartida en su totalidad por Pachón y Garzón (2016, p. 43), Salamanca et al. (2016, p. 5), Caicedo et al. (2016, p. 13), Tejeda y Del Pozo (2016, p. 13), Chaux y Velásquez (2016, p. 6), Díaz et al. (2016, p. 48), Salas (2017, p. 16) y Martínez (2019, pp. 14-15). En esta línea, el aprendizaje de la cultura de la paz en espacios como la Cátedra requiere del aprendizaje de técnicas de resolución de conflictos basadas en el diálogo, para afrontar la discrepancia 
y como parte de las relaciones sociales, partiendo del supuesto de que la paz es indisociable del respeto de los Derechos Humanos (Salamanca et al., 2016).

Finalmente, la tercera perspectiva sobre la paz y la cultura de la paz se basa en su concepto normativo-constitucional. En Colombia, concretamente, la Constitución Política afirma en su artículo 22 que, "la paz es un derecho y un deber de obligatorio cumplimiento" (República de Colombia, 1991). Esta línea conceptual implica que las personas tienen la responsabilidad de relacionarse de forma pacífica con los demás y ser tratadas de la misma manera, con el propósito de mantener relaciones sociales ajenas a la violencia. Por tanto, la paz sería una vivencia en medio de las relaciones sociales. En esta línea, Álvarez y Marrugo (2016) afirman que la paz no puede ser enseñada ni aprendida en una clase donde el maestro es el actor principal portador de una verdad y de la palabra y los estudiantes se presentan como sujetos pasivos. Por tanto, la metodología de clase sería fundamental para hacer de la paz una experiencia diaria bilateral, social, y no un requerimiento más del currículo escolar. Esta tercera perspectiva es la menos desarrollada en la literatura sobre la Cátedra de la Paz, principalmente porque, si bien se establece que la paz es un derecho y un deber, no hay un desarrollo claro sobre qué se entiende por paz y cómo se percibiría en la vida diaria.

\section{Formación ciudadana para la paz y la democracia en instituciones educativas}

En Colombia hay consenso en que la educación de las futuras generaciones es responsabilidad de las instituciones educativas, la familia y la sociedad. El artículo 4 de la Ley 115 de 1994 "por la cual se expide la ley general de la educación" afirma: "Corresponde al Estado, a la sociedad y a la familia velar por la calidad de la educación y promover el acceso al servicio público educativo, y es responsabilidad de la Nación y de las entidades territoriales, garantizar su cubrimiento" (Congreso de la República, 1994). Esta idea se materializa en la existencia del Ministerio de Educa- ción, que regula para todas las instituciones educativas del país los contenidos y competencias mínimas según el año escolar y la asignatura. Por ejemplo, algunos documentos de obligatorio cumplimiento son los Estándares Básicos de Competencias en Ciencias Sociales, los Estándares Básicos en Competencias Ciudadanas, los Estándares Básicos en Ciencias Naturales, y la misma ley y decreto relacionados con la Cátedra de Paz. Estos lineamientos asumen el principio de la homogeneidad, pues todas las instituciones educativas, sin importar su confesión religiosa, su carácter privado o público o su ubicación en el territorio nacional, deben tratar mínimamente los mismos contenidos y competencias.

Más allá de la minucia sobre cómo el Estado dirige la educación formal de los estudiantes, hay una premisa clara y es que el colegio es el lugar al que se asiste para formarse como ciudadano dentro de una democracia. El artículo 67 de la Constitución Política de Colombia afirma:

La educación es un derecho de la persona y un servicio público que tiene una función social; con ella se busca el acceso al conocimiento, a la ciencia, a la tierra, a la técnica y a los demás bienes y valores de la cultura. La educación formará al colombiano en el respeto a los derechos humanos, a la paz y a la democracia; y en la práctica del trabajo y la recreación, para el mejoramiento cultural, científico, tecnológico y para la protección del ambiente. (1991, p. 18)

Por tanto, la educación colombiana adquiere un carácter no solo académico y moral, sino fundamentalmente político; más concretamente, un carácter de "adoctrinamiento", en el sentido de transmitir un conocimiento, unos valores y unos imaginarios democráticos a los estudiantes, con la esperanza de que sean asumidos como verdad y no sean refutados. Con esto no busco afirmar que se deba dejar de educar en democracia o que educar en democracia sea negativo, lo que preciso es que la educación para la paz requiere de una transmisión de valores 
políticos intencionalmente dados, que no admiten razonamiento en contra, pues son asumidos como bases morales sobre las cuales se construye una sociedad pacífica y respetuosa del otro. En ese sentido, expresiones en contra de la igualdad, los Derechos Humanos y el sistema de votación son contrarias al propósito de la enseñanza de la Cátedra de la Paz y de la educación en general, en Colombia.

Frente a si los colegios deberían formar a sus estudiantes en un sistema político, existen dos visiones contrapuestas. De un lado se encuentra Martha Nussbaum, quien aboga por la expansión de los valores democráticos en las escuelas como contrapeso a una educación para la renta y el capital. En su libro Sin fines de lucro. Por qué la democracia necesita de las humanidades (2010) sustenta la necesidad de formar sistemáticamente a estudiantes en valores de respeto, igualdad, empatía, argumentación, capacidad de reflexión y pensamiento crítico, etc. Para ella, la educación es la base de la existencia del sistema democrático: "Ninguna democracia puede ser estable si no cuenta con el apoyo de ciudadanos educados para ese fin" (p. 29). En la otra orilla de pensamiento se encuentra Hannah Arendt, quien en su ensayo "La crisis en la educación", de la compilación Entre el pasado y el futuro: ocho ejercicios sobre la reflexión política, afirma que una de las razones de la crisis de la educación en la modernidad en Estados Unidos es la instrucción política en las escuelas:

En lo que respecta a la política, desde luego que esto implica un serio equívoco: en lugar de la unión de los iguales para asumir el esfuerzo de la persuasión y evitar el riesgo de un fracaso, se produce una intervención dictatorial, basada en la absoluta superioridad del adulto, y se intenta presentar lo nuevo como un fait accompli, es decir, como si lo nuevo ya existiera. Por esta causa, en Europa, la idea de que quien quiera producir nuevas condiciones debe empezar por los niños, fue monopolizada sobre todo por los movimientos revolucionarios de corte tiránico: cuando llegaron al poder, arrebataban los niños a sus padres y sencillamente los adoctrinaban. La educación no debe tener un papel en la politica, porque en la politica siempre tratamos con personas que ya están educadas. (1996, p. 188)

En la perspectiva de Arendt, la educación para la democracia estaría yendo en contravía de su propio fin, pues sería impartida de forma vertical y sin el respeto al otro, como un interlocutor válido de razonamiento, lo cual genera una cultura política más cercana al servilismo que a la democracia. En la perspectiva de Nussbaum, la democracia no es algo innato y requiere ser aprendida, para garantizar su mantenimiento. En ambas autoras, sin embargo, existe una preocupación importante por hallar las causas y las posibles soluciones a una "crisis" en la educación, teniendo como punto de partida las instituciones educativas de los Estados Unidos de América (EE.UU.), aunque, por supuesto, en dos contextos históricos distantes.

Ahora bien, si la educación en Colombia comparte la perspectiva de Nussbaum, ¿en qué medida coincide la teoría normativa-legal con la práctica en el aula? Desde lo normativo, es el Estado quien tiene la obligación de formar a los futuros ciudadanos, a través de las instituciones educativas. Para Salas, el actor sobre el cual recae la responsabilidad mayor serían los docentes, quienes operan como motor de cambio en sus contextos sociales (2017, p. 15). El ejercicio docente se convierte en una profesión importante, especialmente en el contexto histórico colombiano actual, en donde se busca pasar de formas de relacionamiento social predominantemente violentas a una cultura de paz. Para Montagut:

... uno de los retos de la democracia participativa es crear una sociedad integrada por ciudadanos activos, organizados y preparados para asumir un papel dinámico en la escena politica; individuos a quienes, desde la propia escuela, se les eduque para participar en este sistema político. Educar en la ciudadanía es uno de los propósitos de los currículos en las instituciones del país, exi- 
gencia no sólo de una politica pública, sino requerimiento asociado a la imperiosa necesidad de construir en las escuelas culturas que permitan relacionar la ciudadanía con la construcción y permanencia en la democracia y para la democracia, en cuanto hay un reconocimiento del niño y la niña como sujeto de derechos, que se apropia de ellos y asume una postura politica ante su responsabilidad como participante activo en sus comunidades. (2019, p. 99)

Con base en lo anterior, se presentan dos visiones diferentes en la bibliografía. Por un lado, algunos autores argumentan que las instituciones educativas tienen en sus manos una maravillosa oportunidad para transformar a la sociedad desde su campo de acción, mientras que otros se ubican en un escenario más restringido, en el cual los padres y la sociedad dificultan la construcción de ciudadanías de paz, al tener ellos mismos relaciones violentas en familias usualmente disfuncionales.

En la primera línea se encuentran autores como Pachón y Garzón (2016), quienes argumentan que "es precisamente el sistema educativo el medio a través del cual el Estado -como primera instanciainterviene, atiende y establece políticas, en el ámbito estructural, administrativo, metodológico, pedagógico y curricular, entre otras, para dar solución o por lo menos atenuar las consecuencias sociales que esta [la violencia] conlleva" (2016, p. 14). Esta visión es complementada por Díaz, quien afirma que "necesariamente todo cambio social debe estar impulsado por la educación y la paz y Colombia no es ajena a este proceso; si la escuela no es, o no tiene un espacio de formación alrededor de un tema como la paz, ¿qué otra institución social lo podría asumir?" (2016, pp.13-14).

En el segundo grupo, se encuentran autores como Álvarez y Marrugo, quienes expresan que "en principio los padres han de cuidar a sus hijos y los maestros han de construir el conocimiento en el aula con sus alumnos. Entristece ver cómo algunos hogares y algunas escuelas han perdido 'el espíritu de la educación'” (2016, p. 173). Esta misma línea argumental es compartida por Forero : "La institución familiar no puede continuar ausente de los procesos de enseñanza-aprendizaje. El docente está en la obligación de sumirse como agente de aprendizajes, comprometido con los aprendices, con vocación y deseo de transformación, para que así pueda generar espacios hacia la participación, el diálogo y la reflexión" (2018, p. 159)

Más allá de estas dos visiones contrapuestas, la literatura de la Cátedra de la Paz entre 2014 y 2019 caracteriza a las instituciones educativas como un lugar en el cual los estudiantes socializan con sus pares durante gran cantidad de tiempo al día, lo cual hace del colegio un lugar de aprendizaje por excelencia de las relaciones de paz. Si bien los contextos sociales, culturales, políticos y económicos de los estudiantes pueden dificultar la labor de educación para la paz, también es cierto que es precisamente en estos contextos donde más progresos pueden verse en los estudiantes y donde se han puesto los esfuerzos humanos e institucionales. Por tanto, la literatura asume los límites y posibilidades de los colegios y busca estrategias concretas y efectivas para transformar ciudadanos.

\section{Cátedra de Paz en el Aula: un balance pedagógico}

La literatura sobre la Cátedra de Paz ha presentado varios modelos pedagógicos y diversas actividades de clase que buscan ser una guía para que los docentes y estudiantes del país logren aplicar en la realidad lo expuesto en la normativa. Para comprender las propuestas de las fuentes tratadas, se hace necesario analizar cuál es el trasfondo pedagógico en el cual se basan. Caracterizar su visión del rol del docente y del estudiante en el proceso educativo es fundamental para examinar qué tipo de actividades se proponen y cómo se busca alcanzar la educación para la paz. 
En primer lugar, durante la mayor parte del siglo XX se entendió que la escuela era el lugar en el cual las personas aprendían de un sujeto que poseía la verdad y la legitimidad para enseñarla. Así, los docentes eran aquellas personas "sabias" que tenían una "verdad única" que era transmitida unilateralmente a los estudiantes, cuya responsabilidad se limitaba a escuchar atentamente y memorizar los contenidos para realizar exámenes que demostraran su capacidad de repetición. Este enfoque pedagógico es llamado "conductismo" y entiende al aprendizaje como la retención de nuevas conductas o comportamientos a través de estímulos positivos y negativos dados por el docente al estudiante. Por tanto, las actividades de clase estaban centradas en el docente, al realizar exposiciones magistrales, dictados, copias textuales, repeticiones sistemáticas de actividades, como series de planas sobre la palabra escrita erróneamente, o bien castigos "ejemplarizantes" que relacionaran en el estudiante el "error" con sentimientos negativos, como humillación, cansancio o, incluso, dolor.

Sin embargo, la perspectiva conductista de la educación ha venido siendo reemplazada por la visión constructivista, con cambios gigantescos en la educación y la formación de estudiantes. Para el constructivismo, el docente es un acompañante en el proceso de aprendizaje. Así, las clases no se centran en mostrar una verdad única del docente, sino que constituyen un proceso multilateral en el cual los estudiantes aprenden de este y de otros estudiantes, donde el docente no es portador de una verdad absoluta. De hecho, él mismo aprende del conocimiento previo de los estudiantes. Luego aprender no es una actividad limitada al espacio escolar, sino a toda la serie de interacciones que tienen los seres humanos. En el constructivismo, el aprendizaje es la capacidad de los estudiantes para aplicar conocimientos en situaciones cotidianas. Así, las actividades de clase se diferencian drásticamente de aquellas del conductismo, al generar trabajos en equipo, debates, juego de roles, solución conjunta de problemas reales, investigación realizada por los es- tudiantes, mapas conceptuales, actividades de autoevaluación y coevaluación, lluvias de ideas, representaciones gráficas, bitácoras, videos, entre otros. Para Huertas et al., esta perspectiva se concreta en la Cátedra de Paz, pues:

Si se fundamenta la teoría pedagógica contemporánea en la corriente crítica, el impacto recae sobre el currículo que requiere de una actitud reflexiva del maestro dispuesto a integrar sus conocimientos previos con la realidad de sus estudiantes, en el que los educandos se reconozcan entre ellos y se formen como unidad y ante los conflictos tengan la capacidad de resolverlos constructivamente en un modelo cultural referido a la elaboración de una cultura de paz; esto quiere decir que el individuo se construya a si mismo desde su identificación y la de su entorno para la conformación de una cultura que a través del lenguaje comunique a otros lo que significa la convivencia. (2018, p. 79)

La visión constructivista de la educación ha venido siendo implementada por el Ministerio de Educación y los docentes del país basando el aprendizaje y la evaluación en competencias, más que en contenidos. Para las Ciencias Sociales, existen los Estándares Básicos en Ciencias Sociales y los Estándares Básicos en Competencias Ciudadanas, en todos los grados de educación primaria y secundaria del país. Así, la Cátedra de la Paz entra en un contexto de transición entre los modelos pedagógicos conductistas y los constructivistas y se constituye entonces en un reto de aplicación para los docentes del país.

La literatura sobre la Cátedra de la Paz entre 2014 y 2019 se basa en modelos constructivistas, entendiendo que la enseñanza de la paz es vivencial y que estudiantes y docentes están llamados a la transformación de la sociedad en una respetuosa de los Derechos Humanos, pacífica y ambientalmente amigable. Para León y Mejía, una de las actividades de clase en que aplican las habilidades para una ciudadanía en paz es la mediación, como mecanismo alternativo de solución de conflictos, el cual: 
... desarrolla en los estudiantes competencias ciudadanas como el diálogo, la asertividad, la tolerancia, la comprensión y el respeto, que lo forman en el saber ser, saber hacer, para responder de manera ética y objetiva en el ámbito escolary comunitario del que hace parte, buscando que a través de ella se convierta en mediador, transformador y gestor de cambio. (2016, p. 48)

Esta concepción es semejante a la de Carmona Rincón (2016), Caicedo et al. (2016), Chaux \& Velásquez (2016), Chaux et al. (2016), los cuales proponen desarrollar estudios de caso o juegos de roles en los cuales se resuelvan situaciones problemáticas reales o imaginarias, al aplicar la tolerancia, el respeto, el manejo asertivo de emociones y el diálogo. Otras metodologías de clase basadas en el modelo pedagógico constructivista son sugeridas por los autores y señaladas en la Tabla 1.
Si bien las fuentes dan algunas ideas sobre qué tipo de actividades pueden ser usadas para cada clase según los propósitos de la Cátedra, estas no logran concretar cómo realizarlas con coherencia, ni cómo relacionarlas con los propósitos de cada clase dedicada a la Cátedra de la Paz. Es decir, plantean actividades generales que pueden ser útiles, pero que no están articuladas a los objetivos de la Cátedra ni a los Estándares Básicos de Ciencias Sociales y Competencias Ciudadanas del Ministerio de Educación, base de los currículos de formación ciudadana. No obstante, los trabajos Orientaciones generales para la implementación de la Cátedra de la Paz en los establecimientos educativos de preescolar, básica y media de Colombia (Chaux y Velásquez, 2016), Propuesta de desempeños de educación para la paz para ser enriquecidas por los docentes de Colombia (Chaux et al., 2016a) y Secuencias didácticas de Educación para la

Tabla 1. Metodologías de clase propuestas por estudios sobre la Cátedra de la Paz (2014-2019)

\begin{tabular}{|c|c|}
\hline Fuente & Metodologias de clase \\
\hline $\begin{array}{l}\text { León y Mejía } \\
\text { (2016) }\end{array}$ & Explicaciones del docente, trabajo en grupos, aprendizaje basado en problemas, juegos de roles. \\
\hline $\begin{array}{l}\text { Salamanca et al. } \\
\text { (2016) }\end{array}$ & $\begin{array}{l}\text { Actividades basadas en resolución de problemas reales, investigación de los estudiantes, exposiciones de } \\
\text { los estudiantes, bitácoras de experiencias y de trabajos de clase. }\end{array}$ \\
\hline Díaz (2016) & $\begin{array}{l}\text { Exposiciones de los estudiantes, actividades de autoenseñanza, creación de representaciones visuales, } \\
\text { analogías, construcción de preguntas, elaboración de textos y mapas conceptuales. }\end{array}$ \\
\hline Carmona (2016) & $\begin{array}{l}\text { Revisión de documentos, lluvia de ideas colectivas e individuales, creación de murales y representaciones } \\
\text { gráficas del conflicto, observación y análisis de videos, creación de estudios de caso, análisis de caricaturas, } \\
\text { escritura de bitácoras del conflicto escolar. }\end{array}$ \\
\hline $\begin{array}{l}\text { Caicedo et al. } \\
\qquad(2016)\end{array}$ & $\begin{array}{l}\text { Exposiciones estéticas, juegos de roles, cuentos, dibujos y canciones, actividades de perdón colectivo entre } \\
\text { estudiantes y docentes, jornadas de vacunación simbólica contra la violencia, pedidos al país por acciones } \\
\text { de paz a través de cartas de los estudiantes, simulación de los efectos de la violencia y el acoso escolar en } \\
\text { un muñeco de icopor que no retoma a su forma original luego de daños. }\end{array}$ \\
\hline $\begin{array}{l}\text { Chaux et al. } \\
\qquad(2016)\end{array}$ & Generar debates, hacer juegos de roles y discutir dilemas morales. \\
\hline $\begin{array}{l}\text { Chaux et al. } \\
\qquad(2016)\end{array}$ & $\begin{array}{l}\text { GRADO 6: Buscar en internet, trabajar en grupos, analizar casos, generar acciones de impacto, identificar } \\
\text { conflictos, analizar las consecuencias de los actos, reflexionar conjuntamente. } \\
\text { GRADO 7: Construir ejemplos positivos de conflictos que fueron resueltos exitosamente, hacer juegos de } \\
\text { roles y lanzar lluvias de ideas de posibles soluciones, construir preguntas provocadoras, aprender roles de } \\
\text { mediación, crear retroalimentaciones entre estudiantes y hacer debates. } \\
\text { GRADO 8: Construir obras literarias, música, chistes, dichos populares, analizar justificaciones y creencias, } \\
\text { comprender relatos reales. } \\
\text { GRADO 9: Analizar obras populares y casos hipotéticos, identificar tipos ideales de ciudadano, evaluar el rol } \\
\text { de las emociones en la solución de conflictos, reconocer situaciones de violencia. }\end{array}$ \\
\hline
\end{tabular}

Fuente: Elaboración propia con base en las fuentes mencionadas. 
paz para ser enriquecidas por los docentes de Colombia (Chaux et al. 2016b), son importantes, pues generan contenidos, estándares y actividades concretas de clase para cada grado y nivel de desarrollo cognitivo y afectivo de los estudiantes.

\section{Hacia una crítica de la producción académica sobre la Cátedra de Paz: ¿con qué jóvenes nos encontramos en el aula?}

La producción bibliográfica sobre la Cátedra de la Paz se ha analizado hasta este punto en referencia a lo que ofrece, a las diferentes definiciones de paz y al rol que la Cátedra les asigna a las instituciones educativas en la formación de ciudadanos en democracia, y se han detallado las diversas metodologías y enfoques pedagógicos sugeridos para la enseñanza de sus contenidos. Sin embargo, la bibliografía sobre la Cátedra de la Paz no señala las características propias de la generación actual de jóvenes y los retos concretos que afrontan hoy en día. Este vacío en la caracterización de la sociedad hace superfluo cualquier esfuerzo por construir algo diferente, pues, si no se conoce el punto de partida, cómo se puede pensar en la forma de pasar de una ciudadanía conflictiva inicial a una ciudadanía en paz. El afirmar que hay conflictos en una sociedad es tan evidente que sobra decirlo; lo importante es caracterizarlos en detalle: ¿cuáles son los principales retos que afrontan los adolescentes en Colombia?, ¿qué adolescentes se tienen en el aula?, ¿con qué pensamientos, sentimientos e ideas llegan al salón de clases? Uno de los hechos históricos que más han marcado a la sociedad colombiana es el narcotráfico y los diferentes enfrentamientos armados.

El auge del narcotráfico en Colombia a finales del siglo XX dejó una marca imborrable de violencia que Han define como "ese desgarro que no da lugar a ninguna mediación ni reconciliación" (2017b, p. 102). La ruptura del tejido social vino acompañada de la instauración de unos valores que perduraron más allá de lo ilegal, a saber, la idea de que el dinero es el propósito de la vida, de que se trabaja para ganar dinero y se vive para gastarlo, y mientras más pronto sea mejor. ${ }^{4}$ Esta visión fue fomentada por el narcotráfico en un contexto histórico de una economía de consumo. En estos temas, Estanislao Zuleta afirmaba que

... la pobreza y la impotencia de la imaginación nunca se manifiestan de una manera tan clara como cuando se trata de imaginar la felicidad. Entonces comenzamos a inventar paraisos, islas afortunadas, paises de Cucaña. Una vida sin riesgos, sin lucha, sin búsqueda de superación y sin muerte. [...] Todas estas fantasías serían inocentes e inocuas, si no fuera porque constituyen el modelo de nuestros propósitos y de nuestros anhelos en la vida práctica. (1980, p.13)

En Colombia, la felicidad suele relacionarse como la capacidad de gasto. Se anhela poder comprar, y se imagina que la capacidad económica eliminará los riesgos y la lucha y se vivirá en una "isla afortunada". Por tanto, "la histeria de la acumulación y del crecimiento y el miedo a la muerte se condicionan mutuamente. El capital se puede interpretar como tiempo condensado, pues el dinero permite hacer que otros trabajen para uno. El capital infinito genera la ilusión de un tiempo infinito" (Han, 2017b, p. 36). Lo que introdujo el narcotráfico no fue el gusto por el comercio, sino la idea de que el dinero podría venir con mayor rapidez y en cantidades nunca antes vistas.

Los jóvenes de Colombia, sin importar su condición económica, se ven inmersos en la lógica del dinero inmediato. En el plano legal, sus ídolos van desde cantantes de reguetón y música popular que han consolidado un emporio económico en cuestión de meses, hasta youtubers e influencers que hacen su parte en medios digitales. En el plano ilegal, sus

4 Alonso Salazar escribe en su obra No nacimos p'a semilla. La cultura de las bandas juveniles en Medellín 2018): "En la raíz de esa violencia masiva de los jóvenes están factores estructurales de exclusión económica y simbólica, y procesos culturales complejos en los que se ligan al mismo tiempo valores arcaicos y procesos consumistas". 
ídolos son los líderes de los grupos armados, que mezclan poder económico con poder militar y que generan miedo en sus comunidades, asociado erróneamente al respeto.

La sociedad colombiana suele medir el éxito de una persona en función de su capacidad económica, y esta idea es transversal a la percepción general sobre el rol de las instituciones educativas. El colegio puede ser interpretado como el lugar en el cual se buscaría una educación que permita a los jóvenes "salir adelante", lo cual suele relacionarse con mejorar la situación económica propia y de la familia. También es el lugar en el cual los estudiantes permanecen una buena porción del día, evitando su contacto con los "peligros de la calle", y frecuentemente se lo percibe como el medio para lograr un título académico básico que permita o bien trabajar y "producir" o bien ingresar a la educación superior, vista esta como una inversión que será rápidamente retribuida al ingresar al mundo laboral. A su vez, en los sectores económicos superiores, la educación escolar es el lugar donde se adquieren ventajas comparativas sobre otros futuros ciudadanos 5 . Esta visión de la educación como formación para la producción económica es el punto de partida del gobierno nacional. Por ejemplo, en el año 2019, el presidente Iván Duque afirmaba: "Tenemos retos. Por ejemplo, el de la productividad como usted lo decía. Ese reto lo estamos afrontando desde la educación y la formación para el empleo" (citado en Zarmiento, 2019).

La "mentalidad del éxito" es universal. Martha Nussbaum, en su libro Sin fines de lucro. Por qué la democracia necesita de las humanidades (2010), alerta precisamente sobre el auge de lo que ella llama "educación para la renta", una educación preocupada por crear ciudadanos productivos. En esa lógica, no es extraño que la pregunta que ronde el cerebro

$5 \quad$ Algunas ventajas comparativas son: la enseñanza de varios idiomas, altos niveles de matemáticas y tecnología, habilidades para el emprendimiento, actividades de tiempo libre, deportes de alto rendimiento, desarrollo artístico y musical, entre otras. adolescente en relación con sus contenidos de clase sea: "Y esto, ¿para qué me va a servir?". Si se observa el mundo desde la lógica del mercado, es claro que muy pocas cosas sirven.

La añoranza por el dinero como sinónimo de felicidad y la idea de que puede ser alcanzado de forma rápida y sin esfuerzo genera una dislocación de los valores, a los que llamaré por ahora "valores tradicionales". Por tanto, el respeto por el otro, la confianza, la honestidad y la solidaridad se ven sometidos a la ganancia de dinero o, en su expresión más concreta, a la tenencia de algún tipo de propiedad, que bien puede ser un celular, un aparato para escuchar música, unos audífonos, incluso, en sentido amplio, la "propiedad" sobre un amigo o la pareja sentimental. En nuestra sociedad, el irrespeto a los valores tradicionales, en un inicio, se justifica todo el tiempo como una "mentira piadosa", y con el tiempo se naturaliza y termina consolidándose como una forma social de relacionamiento que es promovida casi como un requisito para alcanzar la propia felicidad.

La idea del consumo como principio rector de la sociedad, como medida del éxito y la felicidad, no solo se reduce a la acumulación de dinero, sino que logra modificar las relaciones sociales, específicamente en la instrumentalización del otro en virtud del fin propio (dejando de lado las muchas excepciones que puedan existir en una sociedad en las cuales, claramente, también existe cooperación, amor, amistad, entre otros). Esta actitud se materializa en el adolescente, entre otros aspectos, cuando busca tener más amigos en redes sociales para poder cumplir su propósito de aceptación social, cuando somete a otros a través del acoso escolar con el fin de mostrarse como poseedor durante el tiempo escolar y cuando acumula parejas sentimentales en la construcción de su propia identidad. La instrumentalización de los demás, si bien puede haberse dado en la historia de la humanidad, es hoy en día un valor fomentado en la cultura del país y tiene consecuencias nefastas, como la disminución en la capacidad 
empática de los jóvenes. Para una mentalidad que, en busca de un fin, deja de lado los medios y se guía por lógicas de éxito a través de la acumulación, los sentimientos del otro, los pensamientos del otro, la dignidad del otro no son prioridad. Sobre esta idea de empatía y reciprocidad, Nussbaum recalca la importancia de desarrollar en los niños la capacidad de empatizar, como base de la democracia:

Los niños que desarrollan la capacidad de la comprensión, en muchos casos mediante la experiencia empática, saben comprender el efecto que tienen sus agresiones sobre las otras personas, por quienes sienten cada vez más afecto. Asi, surge el sentimiento de culpa ante la propia agresión y el interés genuino por el bienestar del otro. Si bien la empatía no equivale a la moral, puede proporcionarle ciertos elementos esenciales. A medida que se va formando la capacidad de interés por el otro, aumenta el deseo de controlar la propia agresividad. El niño reconoce que los otros seres no son sus esclavos, sino que son personas separadas con derecho a vivir su vida. (2010, p. 64)

El bajo desarrollo de la empatía genera relaciones menos significativas con los pares, pues, si el otro puede ser instrumentalizado y puede instrumentalizarme, mejor será no fiarse de los demás. Y si bien no sucede en todos los casos, y es claro que hay amistades adolescentes que son de admirar, la tendencia es que estos tengan cada vez menos relaciones significativas con sus pares. Así, la inseguridad propia del adolescente se mezcla con la soledad profunda, entendida esta como falta de conexión, no como aislamiento físico de los demás:

Esa falta de autoestima que es la causante de autolesiones, lo que se da en llamar conducta autolesiva, apunta a una crisis general de gratificación en nuestra sociedad. Yo no puedo producir por mí mismo el sentimiento de autoestima. En efecto, el otro me resulta imprescindible en cuanto instancia de gratificación que me ama, me encomia, me reconoce y me aprecia. El aislamiento narcisista del hombre, la instrumentalización del otro y la competencia total destruyen el clima de gratificación. Desaparece la mirada que confirma y reconoce. (Han, 2017a, p.42)

El relacionamiento express entre los jóvenes lleva a un resquebrajamiento de la comunidad educativa. Los estudiantes no logran conectarse entre sí, ni con sus docentes, al alejarse del autorreconocimiento a través de su apoyo en los demás. Esto genera soledad, que aumenta cada vez más con la existencia de redes sociales que facilitan comunicaciones simples, rápidas, con menos palabras, en menos tiempo y con personas con las cuales no se comparten espacios de forma física. Esta mezcla de situaciones aislantes deviene en la reducción de entornos protectores para los adolescentes y facilita aún más los contextos de violencia, reclutamiento forzoso, presencia de grupos armados, confrontaciones bélicas, pobreza, desigualdad, acoso escolar, porte de armas, consumo de estupefacientes y demás situaciones que pueden presentarse en las instituciones educativas de la ciudad.

En sintesis, las instituciones educativas son percibidas como las encargadas de formar a los futuros ciudadanos en valores y en contenidos. Esta tarea recae principalmente en los docentes, quienes son percibidos por la sociedad y por la literatura de la Cátedra de la Paz como agentes transformadores. Si bien la bibliografía analiza el rol de las instituciones educativas en la formación de ciudadanías de paz, ningún análisis se hace respecto de los estudiantes de hoy en día en Colombia acerca de sus retos, sus perspectivas, los problemas históricos, sus imaginarios sobre la paz, el éxito, entre otros. Así, la bibliografía sobre la Cátedra de la Paz requiere de estudios psicológicos, sociológicos, políticos, sociales sobre los niños y adolescentes de Colombia, que permitan tener un diagnóstico claro sobre cuáles son los problemas de convivencia por afrontar. 


\section{Conclusiones}

La Cátedra de la Paz fue creada en el año 2014 a través de la Ley 1732 y fue reglamentada un año después por medio del Decreto 1038 de 2015. Su objetivo es "crear y consolidar un espacio para el aprendizaje, la reflexión y el diálogo sobre la cultura de la paz y el desarrollo sostenible que contribuya al bienestar general y el mejoramiento de la calidad de vida de la población" (Congreso de la República, 2014). Por ello, se estableció como un espacio académico obligatorio para todas las instituciones educativas del país. La presente revisión documental analizó la producción bibliográfica escrita sobre la Cátedra de la Paz entre 2014 y 2019, enfocada en los contenidos, a la luz de tres preguntas principales: ¿cómo se define la paz?, ¿qué rol se les asigna a las instituciones educativas en la formación de ciudadanías de paz? y ¿cuáles son las metodologías de enseñanza que permitirían la aplicación de los principios y contenidos de la Cátedra? El artículo estuvo dividido en cuatro grandes capítulos de contenido, siguiendo el desarrollo de estas preguntas y sumado a una reflexión sobre los adolescentes en la actualidad colombiana.

La revisión y el análisis permite concluir que la paz y la educación para la paz es entendida como la formación en valores, ideas, comportamientos y actitudes respetuosos de los Derechos Humanos y conscientes de la importancia de la resolución pacífica de los conflictos. En esta línea, se definió paz en tres sentidos. Primero, se mostró la conceptualización de Galtung como paz positiva y paz negativa. Segundo, la paz fue abordada según los lineamientos de la Organización de Naciones Unidas y sus diversos órganos y organismos. Finalmente, fue concebida como un derecho y un deber de obligatorio cumplimiento. La literatura entre 2014 y 2019 fue mayormente cuidadosa en definir el concepto, pues a partir de este se desarrollaron las diferentes explicaciones y teorías de cada autor.

Ahora bien, para alcanzar el objetivo de desarrollar ciudadanías en paz, las fuentes analizadas propusieron varias actividades de clase para aplicar la educación para la paz en el aula. Todas ellas, basadas en la concepción constructivista de la educación, según la cual los docentes no son portadores únicos de conocimiento y el aprendizaje se construye en múltiples sentidos (de estudiante a docente, de estudiante a estudiante y de docente a estudiante). Así, muchas de las actividades son centradas en el estudiante y su papel transformador en la sociedad, dejando de lado las opciones conductistas, donde el estudiante es un ente pasivo-receptivo a las instrucciones y comentarios del docente.

Este apartado es especialmente importante, pues permite comprender que las ciudadanías para la paz solo pueden ser desarrolladas a partir de metodologías que sean democráticas en sí mismas y que permitan la aplicación de conocimientos en la vida diaria de los estudiantes. Por tanto, se encontró coherencia entre los fines educativos de la Cátedra de la Paz y los medios educativos para lograrlo en el tiempo y espacio de las aulas de clase. No obstante, las fuentes requieren de mayor desarrollo metodológico y mayor articulación con los contenidos ya dispuestos en los Estándares Básicos de Competencias en Ciencias Sociales y Competencias Ciudadanas, con el propósito de hacer el aprendizaje más natural y más significativo para los estudiantes, ahorrando recursos humanos y facilitando la aprehensión de competencias.

Finalmente, la bibliografía comprende las instituciones educativas como entidades encargadas de fomentar la construcción de ciudadanías de paz y como promotores principales de cambio en la sociedad y las familias o círculos cercanos de los estudiantes. Así, se comparte la responsabilidad en la formación de los menores, pero el proceso se lidera desde el espacio de socialización por excelencia: el colegio.

En futuras investigaciones se hace necesario articular los contenidos y propuestas del Ministerio de Educación y concretarlos en planes de asignatura 
que sean lógicos, coherentes y que se basen en una metodología inherentemente democrática. Para ello, se sugiere ahondar en la estrategia del debate formativo como una de las muchas herramientas para potenciar las habilidades sociales y de paz de los estudiantes y futuros ciudadanos de Colombia.

\section{Referencias}

Alcaldía Mayor de Bogotá (2018). Notas técnicas. Lineamientos. Orientaciones de la implementación de la Cátedra de Paz con enfoque de cultura ciudadana. https://www.compartirpalabramaestra.org/publicacionese-investigaciones/otras-investigaciones/orientaciones-para-la-implementacion-de-la-catedra-de-pazcon-enfoque-de-cultura-ciudadana

Álvarez, L. y Marrugo, A. (2016). Cátedra de paz en Colombia: una mirada que supera la tiza y el tablero. Redipe, 5(9), 168-174. https://revista.redipe.org/index.php/1/article/view/147

Arendt, H. (1996). Entre el pasado y el futuro: ocho ejercicios sobre la reflexión política. Península.

Asamblea General (1999). A RES/53/243. Organización de las Naciones Unidas. http://www.fund-culturadepaz. org/spa/DOCUMENTOS/DECLARACIONES,\%2ORESOLUCIONES/Declaracion_CulturadPaz.pdf

Caicedo Muñoz, S. C., Moncada, C., Silva, C. A., Valero, M. G., Trigueros, S. y Martínez, M. A. (2016). Memorias de convivencia y paz en la ciudad de Cali: Experiencias y reflexiones en instituciones educativas. Universidad de San Buenaventura: http://hdl.handle.net/10819/4768

Carmona Rincón, I. C. (2016). Educación para la paz. [Tesis de Maestría en Educación y Derechos Humanos] Universidad Autónoma Latinoamericana Medellín, Colombia. http://repository.unaula.edu.co:8080/ bitstream/123456789/959/1/unaula_rep_pos_mae_edu_der_2016_educacion_paz.pdf

Chaux, E. y Velásquez, A. M. (2016). Orientaciones generales para la implementación de la Cátedra de la Paz en los establecimientos educativos de preescolar, básica y media de Colombia. [Portal institucional] Colombia Aprende. https://aprende.colombiaaprende.edu.co/ckfinder/userfiles/files/orientacionesedupaz.pdf

Chaux, E., Mejía, J. F., Lleras, J., Guáqueta, D., Bustamante, A., Rodríguez, G., .. Velásquez, A. (2016a). Propuesta de desempeños de Educación para la paz para ser enriquecidas por los docentes de Colombia. [Portal institucional] Colombia Aprende. https://aprende.colombiaaprende.edu.co/ckfinder/userfiles/files/DESEMPENOSEDUCACIONPARALAPAZ.pdf

Chaux, E., Mejía, J. F., Lleras, J., Guáqueta, D., Bustamante, A., Rodríguez, G. I., Pineda, P. A., Ruiz-Silva, A., Valencia, C., Greniez, Ch., García, D., Alvarado S. A. y Velásquez, A M. (2016b). Secuencias didácticas de Educación para la paz para ser enriquecidas por los docentes de Colombia. Ministerio de Educación. https://docplayer. es/17797278-Secuencias-didacticas-de-educacion-para-la-paz.html

Congreso de la República (1994). Ley 115 de Febrero 8 de 1994. Por la cual se expide la ley general de educación. Ministerio de Educación. https://www.mineducacion.gov.co/1621/articles-85906_archivo_pdf.pdf

Congreso de la República (o1 de og de 2014). Ley 1732 de 2014. Sistema Único de Información Normativa. http:// hdl.handle.net/11520/17323 
DANE - Departamento Administrativo Nacional de Estadística (2018). Educación Formal (EDUC). Información 2018. https://www.dane.gov.co/index.php/estadisticas-por-tema/educacion/poblacion-escolarizada/educacion-formal\#informacion-2018-por-departamento

Díaz G., A., Díaz S., K., Marroquín M., A. y Rincón, N. (2016). Propuesta de Cátedra de la Paz desde una pedagogía crítica para la formación de una ciudadanía activa. [Trabajo de grado] Psicología, Facultad de Ciencias Humanas, Universidad Piloto de Colombia, Bogotá. http://polux.unipiloto.edu.co:8080/00003719.pdf

Díaz Fajardo, J. (2016). La memoria de las víctimas: una estrategia de enseñanza de la cátedra de paz desde la voz de los sin voz. [Tesis] Especialización en Educacion, Cultura y Politica, UNAD, Bucaramanga, Colombia. https://repository.unad.edu.co/handle/10596/12192

Editorial Santillana (2015, 26 de mayo). Firma de la ley para implementar la Cátedra de la Paz. Bogotá, Colombia. [Video] YouTube. https://www.youtube.com/watch?v=jcq9xljJTCQ

Forero López, P. N. (2018). Cátedra de la paz: Praxis del discurso. Miradas, 1(1), 144-161. https://revistas.utp.edu.co/ index.php/miradas/article/view/18881

Galtung, J. (1964). An Editorial. Journal of Peace Research, 1, 1-4.

Han, B.-C. (2017a). La expulsión de lo distinto. Herder.

Han, B.-C. (2017b). Topología de la violencia. Herder.

Herrera, M. F. (2019). Colombia tiene 48.258.494 habitantes, según estima el Dane. La FM, 4 de julio. https:// www.lafm.com.co/colombia/colombia-tiene-48-millones-258-mil-habitantes-segun-el-dane

Huertas, O., López, D. y Fonseca, L. (2018). Educando y aprendiendo para la paz. Pedagogía para la paz en el colegio San Francisco, Ciudad Bolívar (Bogotá, D.C., Colombia). Estudios de Derecho, 75(116), 73-104. https:// revistas.udea.edu.co/index.php/red/article/view/334754

León Giraldo, R. D.y Mejía Gallo,J.(2016). Escuelas de paz, una alternativa para la solución de conflictos en el ambiente escolar, a la luz de la ley 1732 de 2014. [Tesis] Facultad de Ciencias Jurídicas, Programa de Derecho, Universidad de Manizales. http://ridum.umanizales.edu.co:8080/xmlui/bitstream/handle/6789/2904/Proyecto\%20de\%2ogrado\%20de\%202016\%20versi\%C3\%B3n\%20final\%202017.pdf?sequence=1\&isAllowed=y

Martínez Santa, J. (2019). La cátedra de la paz: un escenario emergente de praxis educativa en la Universidad Tecnológica de Pereira. Miradas, 1(2), 178-196. https://revistas.utp.edu.co/index.php/miradas/article/ view/22061

Ministerio de Educación Nacional (2004). Estándares Básicos de Competencias Ciudadanas. Formar para la ciudadanía... jsí es posible! Lo que necesitamos saber y saber hacer. Serie Guías No. 6. https://www.mineducacion.gov.co/1621/articles-75768_archivo_pdf.pdf

Ministerio de Educación Nacional (2004). Estándares Básicos de Competencias Ciencias Sociales. Formar en ciencias: ¡El desafío! Lo que necesitamos saber y saber hacer. Serie Guías No. 7. http://eduteka.icesi.edu.co/ pdfdir/MENEstandaresCienciasSociales2004.pdf 
Montagut Orozco, C. (2019). Aportes de la dimensión jurídico-política a la fundamentación de la Cátedra de la Paz. En R. Delgado Salazar, Educar para la paz. Fundamentos para la implementación de la Cátedra de la Paz (pp. 83-104). Pontificia Universidad Javeriana.

Nussbaum, M. C. (2010). Sin fines de lucro. Por qué la democracia necesita de las humanidades. Katz.

ONU - Organización de las Naciones Unidas (1948). Declaración Universal de los Derechos Humanos. https:// www.un.org/es/universal-declaration-human-rights/

ONU - Organización de Naciones Unidas (1945). Carta de las Naciones Unidas. OAS.org. https://www.oas. org/36ag/espanol/doc_referencia/Carta_NU.pdf

Pachón Susa, A. L. y Garzón Leal, L. M. (2016). Diseño de un modelo pedagógico sobre la Cátedra de la paz para los docentes del área de Sociales de la Institución Educativa Policarpa Salavarrieta de Girardot. [Tesis] Especialización en Gerencia de Proyectos, Universidad Minuto de Dios. https://repository.uniminuto.edu/bitstream/handle/10656/4696/TEGPRO\%2OPACHON\%2OSUSA\%2OANA\%2OLILIANA-2016. pdf? sequence $=1 \&$ isAllowed $=y$

Presidencia de la República (2015). Decreto 1038 de 2015. Sistema Único de Información Normativa. http://www. suin-juriscol.gov.co/viewDocument.asp?ruta=Decretos/30019815

Presidencia de la República (1991). Constitución Politica de Colombia. http://wsp.presidencia.gov.co/Normativa/Documents/Constitucion-Politica-Colombia.pdf

Romero-Castillo, E. (2010). Johan Galtung: "Me impresiona la idea de unos Estados Unidos de Latinoamérica". Deutsche Welle, 20 de septiembre. https://www.dw.com/es/johan-galtung-me-impresiona-la-idea-deunos-estados-unidos-de-latinoam\%C3\%Agrica/a-6021716

Salamanca, M., Rodríguez, M., Cruz, J. D., Ovalle, R., Pulido Albarracín, M. Á. y Molano Rojas, A. (2016). Guía para la implementación de la Cátedra de la Paz. Santillana.

Salas Osorio, A. G. (2017). Cátedra de la paz. Estudio de caso sobre el proceso de implementación en una institución educativa de la ciudad de Cali. [Tesis] Maestría en Derechos Humanos y Cultura de Paz, Facultad de Humanidades y Ciencias Sociales, Universidad javeriana, Cali, Colombia. http://vitela.javerianacali.edu. co/handle/11522/8234

Salazar, A. (2018). No nacimos p’a semilla. La cultura de las bandas juveniles en Medellín. Aguilar.

Tejeda García, D. J. y Del Pozo Serrano, F. J. (2016). Necesidades docentes para la implementación de la educación para la paz en el marco de la Cátedra de la Paz. Olhar de Professor, 19(1). http://marcaly c.redaly c.org/ jatsRepo/684/68459625006/68459625006.pdf 
